\title{
Generalized Rabi models: diagonalization in the spin subspace and differential operators of Dunkl type
}

\author{
Alexander Moroz \\ Wave-scattering.com \\ PACS 02.30.Ik - Integrable systems \\ PACS 42.50.Pq - Cavity quantum electrodynamics; micromasers \\ PACS 85.75. $-\mathrm{d}-$ Spintronics
}

\begin{abstract}
A discrete parity $\mathbb{Z}_{2}$ symmetry of a two parameter extension of the quantum Rabi model which smoothly interpolates between the latter and the Jaynes-Cummings model, and of the two-photon and the two-mode quantum Rabi models enables their diagonalization in the spin subspace. A more general statement is that the respective sets of $2 \times 2$ hermitian operators of the Fulton-Gouterman type and those diagonal in the spin subspace are unitary equivalent. The diagonalized representation makes it transparent that any question about integrability and solvability can be addressed only at the level of ordinary differential operators of Dunkl type. Braak's definition of integrability is shown (i) to contradict earlier numerical studies and (ii) to imply that any physically reasonable differential operator of Fulton-Gouterman type is integrable.
\end{abstract}

Introduction. - Any $2 \times 2$ hermitian operator $\hat{H}$ can be expressed in the form

$$
\hat{H}=\sum_{j=0}^{3} h_{j} \sigma_{j}, \quad h_{j}=\frac{1}{2} \operatorname{Tr}\left(\hat{H} \sigma_{j}\right),
$$

where $h_{j}$ 's are one-dimensional operators in a suitable Hilbert space $\mathfrak{H}$ and here and elsewhere the standard representation of the Pauli matrices $\sigma_{j}, j=1,2,3$, is assumed. For the sake of compactness, we set $\sigma_{0}:=\mathbb{1}$ in summation formulas, with $\mathbb{1}$ being the unit matrix. $\hat{H}$ is said to be of the Fulton-Gouterman type 1, and denoted by $\hat{H}_{F G}$, if (i) $\hat{H}$ is similar to

$$
\hat{H}_{F G}=A \mathbb{1}+B \sigma_{1}+C \sigma_{2}+D \sigma_{3},
$$

and (ii) there is a hermitian operator $\hat{\mathrm{R}}$ such that

$$
[\hat{\mathrm{R}}, A]=[\hat{\mathrm{R}}, B]=0, \quad\{\hat{\mathrm{R}}, C\}=\{\hat{\mathrm{R}}, D\}=0,
$$

where $[$,$] and \{$,$\} denote the conventional commutator and$ anticommutator. (Our definition of $\hat{H}_{F G}$ is broader than the original one by including the term $C \sigma_{2}(c f . \hat{H}$ in eq. (4.1) of ref. [1]).)

A prominent example of the Fulton-Gouterman type Hamiltonians will be shown to be the generalized Rabi model (GRM) studied by Müller et al. 2, 3, Schiró et al. 4], Gritsev et al. [5, 6, and others [7,

$$
\hat{\mathrm{H}}_{\mathrm{gR}}=\gamma a^{\dagger} a+\mu \sigma_{3}+k_{1}\left(a^{\dagger} \sigma_{-}+a \sigma_{+}\right)+k_{2}\left(a^{\dagger} \sigma_{+}+a \sigma_{-}\right),
$$

and the two-photon (TPRM) and the two-mode quantum Rabi models (TMRM) 8 [ [cf. eq. (9) below]. Here $\hat{a}$ and $\hat{a}^{\dagger}$ are the conventional boson annihilation and creation operators satisfying commutation relation $\left[\hat{a}, \hat{a}^{\dagger}\right]=1$. In the Fock-Bargmann representation [9, 10,

$$
a \rightarrow \mathrm{d} / \mathrm{d} z=\mathrm{d}_{z}, \quad a^{\dagger} \rightarrow z,
$$

$\hat{\mathrm{H}}_{\mathrm{gR}}$ becomes a first-order differential operator on $\mathfrak{B} \otimes \mathbb{C}^{2}$, where $\mathfrak{B}$ is the Fock-Bargmann Hilbert space of entire analytic functions isomorphic to $L^{2}(\mathbb{R})[9$. The GRM interpolates between the Jaynes-Cummings model (JCM) [1] (for $k_{2}=0$ ) and the original quantum Rabi model (RM) 12 22 (for $k_{1}=k_{2}=k$ ). The RM describes the simplest interaction between a cavity mode with a bare frequency $\omega$ and a two-level system, or a qubit, with a bare resonance frequency $\omega_{0}$. One has $\gamma=\hbar \omega>0$, $k_{1}=k_{2}=\hbar g>0$, with $g$ being a coupling constant, $\mu=\hbar \omega_{0} / 2$, where $\hbar$ is the reduced Planck constant. The RM with a negative sign of its parameters $g$ and $\mu$ ( $c f$. eq. (12) of ref. 14]) is used to describe an excitation hopping between two sites ( $\mu$ is then a tunneling parameter) and is relevant in understanding the transition between untrapped and trapped behavior of an exciton. The GRM serves as a non-trivial model in spin resonance, for various problems involving the interaction between electronic and vibrational degrees of freedom in molecules and solids, and in quantum optics 23 27. The RM and GRM are 
presently the focus of intense experimental and theoretical activity for cavity- and circuit-QED setups, superconducting q-bits, nitrogen vacancy centers, etc. [6, 23, 27]. With new experiments rapidly approaching the limit of the socalled deep strong coupling regime, there is every reason to believe that such systems could open up a rich vein of research on truly quantum effects with implications for quantum information science and fundamental quantum optics. There are several further motivations to consider this model. It can be mapped onto the model describing a two-dimensional electron gas with Rashba $\left(\alpha_{R} \sim k_{1}\right)$ and Dresselhaus $\left(\alpha_{D} \sim k_{2}\right)$ spin-orbit couplings subject to a perpendicular magnetic field (the Zeeman splitting thereby equals $2 \mu$ ) [5, 6, 28. The Rashba spin-orbit coupling (SOC) can be tuned by an applied electric field while the Zeeman term is tuned by an applied magnetic field. This allows us to explore the whole parameter space of the model. In ref. 29] a possible realization of tunable Rashba and Dresselhaus SOC with ultracold alkali atoms is proposed, where each state is coupled by a two-photon Raman transition. Further examples of physical realizations of the GRM include (i) electric-magnetic coupling of light and matter, and (ii) effective realization of the model using 3 - and 4-level emitters [6].

Provided that $\hat{\mathrm{R}}^{2}=1$, any $\hat{H}_{F G}$ enjoys a discrete $\mathbb{Z}_{2}$ symmetry $\hat{\Pi}_{F G}=\hat{\mathrm{R}} \sigma_{1}:\left[\hat{H}_{F G}, \hat{\Pi}_{F G}\right]=0, \hat{\Pi}_{F G}^{2}=1$. The $\mathbb{Z}_{2}$-symmetry suffices to partially diagonalize $\hat{H}_{F G}$ operating on the Hilbert space $\mathfrak{B} \otimes \mathbb{C}^{2}$ in the spin subspace 30. Indeed, a sufficient condition for the spin-subspace diagonalization of a Hamiltonian $\hat{H}$ on $\mathfrak{B} \otimes \mathbb{C}^{N}$ is that $\hat{H}$ possesses an Abelian symmetry $G$ of the order $N$. Surprisingly enough, the diagonalization of the GRM, TPRM, and TMRM in the spin subspace has not been discussed yet - $c f$. refs. 2, 8, 12, 13, 15, 17, 22, - even though the diagonalization can be performed by rather straightforward unitary transformation:

Theorem 1: Any $\hat{H}_{F G}$ given by (2) can be diagonalized in the spin subspace by means of a unitary transformation,

$$
\begin{aligned}
U_{F G} \hat{H}_{F G} U_{F G}^{-1} & =(A+D) \mathbb{1}+B \hat{\mathrm{R}} \sigma_{3}-\mathrm{i} C \hat{\mathrm{R}} \sigma_{3}, \\
U_{F G} \hat{\Pi}_{F G} U_{F G}^{-1} & =\sigma_{3},
\end{aligned}
$$

induced by

$$
U_{F G}=\frac{1}{2}\left[(1+\hat{\mathrm{R}}) U_{13}+(1-\hat{\mathrm{R}}) U_{2}^{-1}\right],
$$

where $U_{13}=\left(\sigma_{1}+\sigma_{3}\right) / \sqrt{2}$ and $U_{2}=\left(\mathbb{1}+\mathrm{i} \sigma_{2}\right) / \sqrt{2}$. Thereby an original spin- $1 / 2$ problem in the Hilbert space $\mathfrak{H} \otimes \mathbb{C}^{2}$ decouples into two distinct one-dimensional problems in $\mathfrak{H}$, each characterized by the operator

$$
\hat{\mathrm{L}}_{ \pm}=A+D \pm(B-\mathrm{i} C) \hat{\mathrm{R}},
$$

where the \pm sign corresponds to the respective positive and negative parity eigenspaces.

The letter is organized as follows. Theorem 1 is applied to the GRM, TPRM and TMRM. For the GRM the oper- ators $\hat{\mathrm{L}}_{ \pm}$become first-order ordinary differential operators of Dunkl type 31,32, whereas for the TPRM and TMRM they become second-order differential operators of Dunkl type. The Dunkl type operators, which are characterized in that they contain a reflection operator, became a branch of mathematics only as late as 1990 31. Working in the diagonalized representation makes it transparent that any question about integrability and solvability can be addressed only at the level of ordinary differential operators of Dunkl type. Braak's definition of integrability 18 is shown (i) to imply that any physically reasonable differential operator of Dunkl type is integrable and (ii) to contradict earlier numerical studies by Müller et al. [2, 3].

For completeness an adaption of Wagner's proof directly to $\hat{H}_{F G}$ as given by (2) is provided. The reverse of Wagner's theorem is also shown to be true and the following result is proven:

Theorem 2: A hermitian operator $\hat{H}$ has the FultonGouterman form (2), (3) if and only if $\hat{H}$ is unitary equivalent to an operator diagonal in the spin subspace.

Diagonalization in the spin subspace. - In view of Theorem 1, it suffices to demonstrate the FultonGouterman form of a given Hamiltonian. $\hat{\mathrm{H}}_{\mathrm{gR}}$ can be brought into the Fulton-Gouterman form (2) upon applying a (nonunitary) similarity transformation $\hat{\mathcal{H}}_{\mathrm{gR}}=$ $\mathbf{W} \hat{\mathrm{H}}_{\mathrm{gR}} \mathbf{W}^{-1}$ with

$$
\mathbf{W}=\frac{1}{\sqrt{2}}\left(\begin{array}{rr}
w & w^{-1} \\
w & -w^{-1}
\end{array}\right), \quad \mathbf{W}^{-1}=\frac{1}{\sqrt{2}}\left(\begin{array}{ll}
w^{-1} & w^{-1} \\
w & -w
\end{array}\right),
$$

where $w=\left(k_{2} / k_{1}\right)^{1 / 4}$. Under the similarity transformation

$$
\begin{gathered}
\sigma_{+} \rightarrow \frac{w^{2}}{2}\left(\sigma_{3}-\mathrm{i} \sigma_{2}\right), \quad \sigma_{-} \rightarrow \frac{1}{2 w^{2}}\left(\sigma_{3}+\mathrm{i} \sigma_{2}\right), \\
k_{1} \sigma_{+}+k_{2} \sigma_{-} \rightarrow \sqrt{k_{1} k_{2}} \sigma_{3}, \quad \sigma_{3} \rightarrow \sigma_{1}, \\
k_{2} \sigma_{+}+k_{1} \sigma_{-} \rightarrow \frac{k_{1}^{2}+k_{2}^{2}}{\sqrt{k_{1} k_{2}}} \sigma_{3}+\mathrm{i} \frac{k_{1}^{2}-k_{2}^{2}}{\sqrt{k_{1} k_{2}}} \sigma_{2} .
\end{gathered}
$$

The transformed $\hat{\mathcal{H}}_{\mathrm{gR}}$ divided by $\gamma$ takes on the FultonGouterman form (2) with

$$
A=\hat{a}^{\dagger} \hat{a}, \quad B=\Delta, \quad C=\mathrm{i} \frac{\lambda_{-}}{\kappa} \hat{a}^{\dagger}, \quad D=\kappa a+\frac{\lambda_{+}}{\kappa} \hat{a}^{\dagger},
$$

where

$$
\Delta:=\frac{\mu}{\gamma}, \quad \lambda_{ \pm}:=\frac{k_{1}^{2} \pm k_{2}^{2}}{2 \gamma^{2}}, \quad \kappa:=\frac{\sqrt{k_{1} k_{2}}}{\gamma} .
$$

The Fulton-Gouterman symmetry operation is realized by unitary $\hat{\mathrm{R}}=e^{\mathrm{i} \pi \hat{a}^{\dagger} \hat{a}}$, which induces reflections of the annihilation and creation operators: $\hat{a} \rightarrow-\hat{a}, \hat{a}^{\dagger} \rightarrow-\hat{a}^{\dagger}$, and leaves the boson number operator $\hat{a}^{\dagger} \hat{a}$ invariant [1]. According to Wagner's theorem, $\hat{\mathcal{H}}_{\mathrm{gR}}$ in the Fock-Bargmann representation (5) is unitary equivalent to

$$
\hat{\mathcal{H}}_{\mathrm{gR}}=\left[(z+\kappa) \mathrm{d}_{z}+\frac{\lambda_{+} z}{\kappa}\right]+\left(\frac{\lambda_{-} z}{\kappa}+\Delta\right) \sigma_{3} \hat{\mathrm{R}} .
$$


In the limit $k_{1}=k_{2}=g$ leading to the RM in a unitary equivalent single-mode spin-boson picture:

$$
\begin{gathered}
\lambda_{-}=0, \quad \kappa=\frac{g}{\omega}, \quad \frac{\lambda_{+}}{\kappa}=\kappa, \\
A=z \mathrm{~d}_{z}, \quad B=\Delta, \quad C=0, \quad D=\kappa\left(z+\mathrm{d}_{z}\right),
\end{gathered}
$$

(we set the reduced Planck constant $\hbar=1$ ) and $\mathbf{W}$ becomes the unitary transformation $U_{13}$ [15,20]:

$$
\mathbf{W}=\frac{1}{\sqrt{2}}\left(\begin{array}{rr}
1 & 1 \\
1 & -1
\end{array}\right)=\frac{1}{\sqrt{2}}\left(\sigma_{1}+\sigma_{3}\right) \equiv U_{13}=U_{13}^{-1} .
$$

(A different $\mathbf{W}$ has been used by Gritsev et al. 6 which in its symmetrized form reduces to $\left(\sigma_{1}-\sigma_{3}\right) / \sqrt{2}$ in the limit $k_{1}=k_{2}$.)

The Hamiltonians $\hat{\mathrm{H}}_{2 p}=\omega a^{\dagger} a+\beta \sigma_{3}+g \sigma_{1}\left[\left(a^{\dagger}\right)^{2}+a^{2}\right]$ and $\hat{\mathrm{H}}_{2 m}=\omega\left(a_{1}^{\dagger} a_{1}+a_{2}^{\dagger} a_{2}\right)+\beta \sigma_{3}+g \sigma_{1}\left(a_{1}^{\dagger} a_{2}^{\dagger}+a_{1} a_{2}\right)$ of the TPRM and the TMRM, respectively, are on unitary transforming with $U_{13}$ brought into the FoultonGouterman form ( $c f$. eqs. (4.3) and (5.3) of [8])

$$
\hat{\mathrm{H}}_{2 F G}=\gamma\left(K_{0}-c\right)+\Delta \sigma_{1}+\sigma_{3}\left(K_{+}+K_{-}\right),
$$

where $\gamma=\omega / g, \Delta=\beta / g$. Compared to the Heisenberg algebra of $a$, $a^{\dagger}$ in $\hat{\mathrm{H}}_{\mathrm{gR}}$, the operators $K_{ \pm}, K_{0}$ in (9) form the usual $s u(1,1)$ Lie algebra, $\left[K_{0}, K_{ \pm}\right]= \pm K_{ \pm}$, $\left[K_{+}, K_{-}\right]=-2 K_{0}$. In the case of the TPRM, $c=1 / 4$,

$$
K_{+}=\frac{1}{2}\left(a^{\dagger}\right)^{2}, \quad K_{-}=\frac{1}{2} a^{2}, \quad K_{0}=\frac{1}{2}\left(a^{\dagger} a+\frac{1}{2}\right),
$$

whereas, in the case of the TMRM, $c=1 / 2$, and

$$
K_{+}=a_{1}^{\dagger} a_{2}^{\dagger}, \quad K_{-}=a_{1} a_{2}, \quad K_{0}=\frac{1}{2}\left(a_{1}^{\dagger} a_{1}+a_{2}^{\dagger} a_{2}+1\right) .
$$

The parity symmetry $\hat{\Pi}_{F G}=\hat{\mathrm{R}} \sigma_{1}$ is realized by unitary $\hat{\mathrm{R}}=e^{\mathrm{i} \pi K_{0}}$, which induces reflections of $K_{ \pm}$and leaves $K_{0}$ invariant.

The Fock-Bargmann Hilbert space $\mathfrak{B}$ is based on the coherent states associated with the Heisenberg algebra [10. In the present case, $\mathfrak{B}$ gets replaced by a more general Hilbert space of entire analytic functions of growth $(1,1)$ associated with the so-called Barut-Girardello coherent states 10 of the annihilation operator $K_{-}$of the $s u(1,1)$ Lie algebra. In an infinite-dimensional unitary irreducible representation, known as the positive discrete series $\mathcal{D}^{+}(q)$, the operators $K_{ \pm}, K_{0}$ of the TPRM, which realize the single-mode bosonic representation of $s u(1,1)$, are represented as differential operators,

$$
K_{0}=z \mathrm{~d}_{z}+q, \quad K_{+}=z / 2, \quad K_{-}=2 z \mathrm{~d}_{z}^{2}+4 q \mathrm{~d}_{z},
$$

where the parameter $q$, called Bargmann index, satisfies $q=1 / 4,3 / 4$. The operators $K_{ \pm}, K_{0}$ of the TMRM providing the two-mode bosonic representation of $s u(1,1)$ have the single-variable differential realization as

$$
K_{0}=z \mathrm{~d}_{z}+q, \quad K_{+}=z, \quad K_{-}=z \mathrm{~d}_{z}^{2}+2 q \mathrm{~d}_{z},
$$

where $q>0$ can be any integer or half-integer [8,10].
Linear differential operators of Dunkl type. The action of $\hat{\mathrm{R}}$ in the above cases reduces to reflections $\hat{\mathrm{R}} f(z)=f(-z)$ in a suitable Hilbert space of entire analytic functions. In the case of the GRM, the respective diagonal components $\hat{\mathrm{L}}_{ \pm}$defined by (8) become linear first-order ordinary differential operators of Dunkl type,

$$
\hat{\mathrm{L}}_{ \pm}=(z+\kappa) \mathrm{d}_{z}+\frac{\lambda_{+} z}{\kappa} \pm\left(\frac{\lambda_{-} z}{\kappa}+\Delta\right) \hat{\mathrm{R}}
$$

In the limit of the RM one recovers

$$
\hat{\mathrm{L}}_{ \pm}=(z+\kappa) \mathrm{d}_{z}+\kappa z \pm \Delta \hat{\mathrm{R}}
$$

(cf. eq. (21) of ref. [14] and eq. (2.1) of ref. [16]). For the respective TPRM and the TMRM one finds

$$
\begin{gathered}
\hat{\mathrm{L}}_{ \pm ; 2 p}=2 z \mathrm{~d}_{z}^{2}+(4 q+\gamma z) \mathrm{d}_{z}+\frac{z}{2}+\gamma\left(q-\frac{1}{4}\right) \pm \Delta \hat{\mathrm{R}}, \\
\hat{\mathrm{L}}_{ \pm ; 2 m}=z \mathrm{~d}_{z}^{2}+(2 q+\gamma z) \mathrm{d}_{z}+z+\gamma\left(q-\frac{1}{2}\right) \pm \Delta \hat{\mathrm{R}} .
\end{gathered}
$$

For a general $\hat{\mathrm{L}}_{ \pm}$in (8) one has

$$
\left[\hat{\mathrm{L}}_{ \pm}, \hat{\mathrm{R}}\right]=\mp 2 \mathrm{i} C+2 D \hat{\mathrm{R}} \neq 0 .
$$

Therefore also $\left[\hat{\mathrm{L}}_{ \pm}, \hat{\mathrm{R}} \hat{\mathrm{L}}_{ \pm}\right] \neq 0$. Whereas for an eigenvector $\phi$ of $\hat{\mathrm{L}}_{ \pm}$one has $\hat{\mathrm{R}} \hat{\mathrm{L}}_{ \pm} \phi=\epsilon \hat{\mathrm{R}} \phi$, one cannot say anything definite about $\hat{\mathrm{L}}_{ \pm} \hat{\mathrm{R}} \phi$.

Note that in the absence of the reflection operator $\hat{\mathrm{R}}$ in eq. (11), e.g. with $\hat{\mathrm{L}}_{ \pm}=(z+\kappa) \mathrm{d}_{z}+\kappa z \pm \Delta$, the eigenvalue problem,

$$
(\hat{\mathrm{L}}-\epsilon) \phi=0,
$$

can be easily integrated. One finds $\phi(z)=\operatorname{const}(z+$ $\kappa)^{\kappa^{2} \pm \Delta-\epsilon} \mathrm{e}^{-\kappa z}$, where 'const' is an integration constant. The solutions will be holomorphic if and only if $\kappa^{2} \pm \Delta-\epsilon \epsilon$ $\mathbb{N}_{0}$. In spite of a deceptive simplicity of $\hat{\mathrm{L}}_{ \pm}$in eqs. (10), (11), a rigorous analytic solution of (13) remains an unsolved problem (in the sense that analytic expressions for eigenvalues are not known - cf. ref. 33). This demonstrates that the reflection operator $\hat{\mathrm{R}}$ is a highly nontrivial obstruction for solving the eigenvalue problem (13) [32].

Each term of the one-dimensional operator $\hat{\mathrm{L}}_{ \pm}$in (10), (11), (12) does not change the degree of a monomial $z^{n}$ by more than \pm 1 . Thereby the resulting eigenvalue problem (13) naturally reduces to a three-term recurrence relation (TTRR) [8, 13, 17, 19, 20, 34. Thus each $\hat{\mathrm{L}}_{ \pm}$corresponds to the so-called irreducible component of ref. 21]. Those have been shown to have a nondegenerate spectrum under very general conditions, and hence no level crossing while varying coupling parameter(s). Alternatively, the nondegeneracy applies to all problems where the Hamiltonian operator is a self-adjoint extension of a tridiagonal Jacobi matrix of deficiency index $(1,1)$ 17, 35. Therefore, all $\hat{H}_{F G}$ leading to a TTRR have avoided level crossings. The above arguments provide rigorous proof for the avoided crossing observed numerically by Müller et al. 2, 3, 
Braak's definition of integrability. - According to Braak's definition of integrability [18: If each eigenstate of a quantum system with $f_{1}$ discrete and $f_{2}$ continuous degrees of freedom can be uniquely labeled by $f_{1}+f_{2}=f$ quantum numbers $\left\{d_{1}, \ldots, d_{f_{1}}, c_{1}, \ldots, c_{f_{2}}\right\}$, such that the $d_{j}$ can take on $\operatorname{dim}\left(H_{j}\right)$ different values, where $H_{j}$ is the state space of the $j$ th discrete degree of freedom and the $c_{k}$ range from 0 to infinity, then this system is quantum integrable. The RM has $f_{1}=f_{2}=1$ and degeneracies take place only between levels of states with different parity, whereas within the parity subspaces no level crossings occur. The global label for the RM [valid for all values of $\kappa$ in eq. (11)] is two dimensional, with one label for the parity and the other being the energy sorting number within a given parity subspace, and hence, according to Braak, the $\mathrm{RM}$ is quantum integrable. But this leads to an inflation of integrable models, because the avoided level crossings between states of equal parity is generic for the models studied here. Following Braak's arguments, all physically reasonable $\hat{H}_{F G}$ are necessarily quantum integrable.

Braak's arguments appear to be based on a wrong assessment of the role of discrete symmetries. The latter divide the Hilbert space of $\hat{H}_{F G}$ into invariant subspaces. In general, this does not result in symmetry-induced leveldegeneracies, but it does lead to accidental degeneracies between levels belonging to different invariant subspaces (cf. Judd solutions). Such level crossings exist independently of whether or not $\hat{H}_{F G}$ is integrable 3 .

The general rule has always been to analyze corresponding invariant or irreducible components. For instance, in statistical analysis of the eigenvalues of quantum billiards one performs the so-called desymmetrization, which reduces the study to a fundamental domain of a discrete group [36. The diagonalized representation makes it transparent that integrability and solvability of the GRM can be addressed only at the level of first-order one-dimensional differential operators $\hat{\mathrm{L}}_{ \pm}$. The latter necessitates considering each invariant parity subspace independently. In their thorough numerical studies, Müller et al. 2, 3, did just that. They made use of the fact that a quantum integrability cannot be inferred from quantum invariants as simply as classical integrability can be inferred from integrals of the motion (analytic invariants). Commuting operators can always be constructed irrespective of whether the model is (classically) integrable or not 2, 3, 37. When Einstein-Brillouin-Keller quantization is possible, it applies to all conserved dynamical variables (not only to the Hamiltonian) and in particular to the time average of any dynamical variable. Any operator $T$ that is not already an invariant, $[H, T] \neq 0$, can be turned into an invariant via time average. In the energy representation, the time average strips $T$ of all its off-diagonal elements. The resulting operator $I_{T}=\langle T\rangle$ being diagonal in the energy representation thus commutes with $H$ by construction [37. Müller et al. 2, 3] studied two-dimensional patterns of quantum invariants $\left\{\left(\epsilon_{n},\left\langle T_{j}\right\rangle_{n}\right)\right\}$, where $\epsilon_{n}$ is the $n$th eigenvalue, and $T_{1}=a^{\dagger} \sigma_{+}, T_{2}=a^{\dagger}\left(\sigma_{-}+\sigma_{+}\right)$.
(Although $T_{j}$ 's are not hermitian, the matrix elements $\left\langle n\left|T_{j}\right| n\right\rangle=\left\langle T_{j}\right\rangle_{n}$ happen to be real for all energy eigenstates [2.) The patterns of points $\left\{\left(\epsilon_{n},\left\langle T_{j}\right\rangle_{n}\right)\right\}$ in invariant parity subspaces were found to be strikingly different for the respective integrable $\left(k_{1} k_{2}=0\right)$ and nonintegrable $\left(k_{1} k_{2} \neq 0\right)$ cases. A qualitative change in pattern required the assignment of mutually exclusive sets of quantum numbers to the same set of eigenstates in different parameter regimes 2, 3. In the integrable cases, the patterns formed two separate linear strands of points. Level crossings required a two dimensional label for an unambiguous assignment of levels, each label corresponding to one of the respective quantum invariants. Contrary to that, a single level sorting quantum number sufficed to label all eigenstates in the presence of the avoided level crossings between states of equal parity for nonintegrable cases. In contrast to Braak's conclusion, avoided level crossings were found to be the trademark of quantum nonintegrability. The integrable and nonintegrable cases revealed also unambiguously different patterns of coordinated motion of all states with given parity in the plane of invariants $\left(\epsilon_{n},\left\langle T_{2}\right\rangle_{n}\right)$ as the interaction strength (i.e. $\Lambda$ in the parametrization $k_{1}=\Lambda \cos \alpha, k_{2}=\Lambda \sin \alpha$ of the coupling constants) gradually increased [3. The distinctive attributes of quantum invariants in the integrable and nonintegrable regimes of a quantum system are subtle but not ambiguous. As soon as $k_{1} k_{2} \neq 0$ (or $\alpha \neq 0, \pi / 2$ ), the GRM was found nonintegrable [2, 3].

Proof of Theorem 1. - It is expedient to introduce

$$
U_{j k l}=\frac{1}{2}\left[(1+\hat{\mathrm{R}}) U_{j k}+(1-\hat{\mathrm{R}}) U_{l}\right]
$$

with unequal $j, k, l=1,2,3$, where

$$
U_{j k}=\left(\sigma_{j}+\sigma_{k}\right) / \sqrt{2}, \quad U_{l}=\left(\mathbb{1}+\mathrm{i} \sigma_{l}\right) / \sqrt{2},
$$

are $2 \times 2$ unitary matrices. Any $U_{j k l}$ is thus a linear combination of unitary matrices with the coefficients being one-dimensional projectors $\hat{\mathrm{P}}_{ \pm}=(1 \pm \hat{\mathrm{R}}) / 2 . U_{j k l}$ itself is unitary:

$$
\begin{aligned}
U_{j k l} U_{j k l}^{-1}= & \frac{1}{8}\left[(1+\hat{\mathrm{R}}) U_{j k}+(1-\hat{\mathrm{R}}) U_{l}\right] \\
& \times\left[(1+\hat{\mathrm{R}}) U_{j k}+(1-\hat{\mathrm{R}}) U_{l}^{-1}\right] \\
= & \frac{1}{4}\left[(1+\hat{\mathrm{R}})^{2}+(1-\hat{\mathrm{R}})^{2}\right] \mathbb{1} \\
= & \frac{1}{4}[2+2 \hat{\mathrm{R}}+2-2 \hat{\mathrm{R}}] \mathbb{1}=\mathbb{1} .
\end{aligned}
$$

Now,

$$
\begin{aligned}
& U_{j k l} \sigma_{j} U_{j k l}^{-1} \\
& \quad=\frac{1}{4}\left[(1+\hat{\mathrm{R}})^{2} U_{j k} \sigma_{j} U_{j k}+(1-\hat{\mathrm{R}})^{2} U_{l} \sigma_{j} U_{l}^{-1}\right] .
\end{aligned}
$$

For unequal $j, k, l=1,2,3$ one finds

$$
\begin{gathered}
U_{j k} \sigma_{j} U_{j k}=\sigma_{k}, \quad U_{j k} \sigma_{l}=-\sigma_{l} U_{j k}, \quad U_{l} \sigma_{j}=\sigma_{j} U_{l}^{-1}, \\
U_{l} \sigma_{j} U_{l}^{-1}=-\epsilon_{l j k} \sigma_{k}, \quad U_{l}^{-1} \sigma_{j} U_{l}=\epsilon_{l j k} \sigma_{k},
\end{gathered}
$$


where $\epsilon_{l j k}$ is the usual totally antisymmetric Levi-Civita symbol. In arriving at the final results we have repeatedly used

$$
-\mathrm{i} \sigma_{1} \sigma_{2} \sigma_{3}=\mathbb{1}, \quad \sigma_{j} \sigma_{k}=\mathrm{i} \epsilon_{j k l} \sigma_{l} .
$$

Under the action of unitary $U_{l}$ the matrix $\sigma_{l}$ remains invariant. It holds trivially that $U_{j k l} \sigma_{0} U_{j k l}^{-1}=\sigma_{0}$. Given the properties (16), one can verify that (modulo a sign change and multiplication by $\hat{\mathrm{R}}$ ):

$(*)$ the unitary transformation induced by $U_{j k l}$ with unequal $j, k, l=1,2,3$ interchanges $\sigma_{j}$ and $\sigma_{k}$ while leaving $\sigma_{0}$ and $\sigma_{l}$ invariant.

For any operator $\hat{\mathrm{X}}$ on $\mathfrak{H}$ commuting with $\hat{\mathrm{R}}$

$$
U_{j k l} \hat{\mathrm{X}} \sigma_{j} U_{j k l}^{-1}=\hat{\mathrm{X}}\left(U_{j k l} \sigma_{j} U_{j k l}^{-1}\right),
$$

whereas for any operator $\hat{\mathrm{Y}}$ on $\mathfrak{H}$ anticommuting with $\hat{\mathrm{R}}$

$$
U_{j k l} \hat{Y} \sigma_{j} U_{j k l}^{-1}=\left(U_{j k l} \hat{Y} U_{j k l}^{-1}\right)\left(U_{j k l} \sigma_{j} U_{j k l}^{-1}\right) .
$$

Table 1. $U_{F G}=\frac{1}{2}\left[(1+\hat{\mathrm{R}}) U_{13}+(1-\hat{\mathrm{R}}) U_{2}^{-1}\right]$.

\begin{tabular}{|c|c|c|c|c|}
\hline$\hat{\mathrm{O}}$ & $A \mathbb{1}$ & $B \sigma_{1}$ & $C \sigma_{2}$ & $D \sigma_{3}$ \\
\hline$U_{F G} \hat{\mathrm{O}} U_{F G}^{-1}$ & $A \mathbb{1}$ & $B \hat{\mathrm{R}} \sigma_{3}$ & $-\mathrm{i} C \hat{\mathrm{R}} \sigma_{3}$ & $D \mathbb{1}$ \\
\hline
\end{tabular}

The unitary transformation $U_{F G}$ of Theorem 1 is a particular case of $U_{j k l}$ defined by (14). One has $U_{F G}=U_{132}^{-1}=$ $U_{13,-2}$, where the minus sign in front of 2 stands for the inverse of $U_{2}$ in the definition (7). On combining relations (16),

$$
U_{F G} \sigma_{1} U_{F G}^{-1}=\frac{1}{4} \sigma_{3}\left[(1+\hat{\mathrm{R}})^{2}-(1-\hat{\mathrm{R}})^{2}\right]=\hat{\mathrm{R}} \sigma_{3} .
$$

Hence for $A$ and $B$ commuting with $\hat{\mathrm{R}}$ one has $U_{F G} A \sigma_{0} U_{F G}^{-1}=A \sigma_{0}$ and $U_{F G} B \sigma_{1} U_{F G}^{-1}=B \hat{\mathrm{R}} \sigma_{3}$, respectively. With the help of identities (16) one has

$$
\begin{gathered}
U_{F G} \sigma_{0} U_{F G}^{-1}=\sigma_{0}, \quad U_{F G} \sigma_{1} U_{F G}^{-1}=\hat{\mathrm{R}} \sigma_{3}, \\
U_{F G} \sigma_{2} U_{F G}^{-1}=-\hat{\mathrm{R}} \sigma_{2}, \quad U_{F G} \sigma_{3} U_{F G}^{-1}=\sigma_{1},
\end{gathered}
$$

conforming to the general rule $(*)$. Because

$$
\begin{gathered}
U_{j k} U_{l}=U_{l}^{-1} U_{j k}=\frac{1}{2}\left[\sigma_{j}+\sigma_{k}-\epsilon_{j k l}\left(\sigma_{j}-\sigma_{k}\right)\right], \\
\text { i.e., } U_{13} U_{2}=U_{2}^{-1} U_{13}=\sigma_{1} \text {, one has } \\
U_{F G} \hat{\mathrm{Y}} U_{F G}^{-1}=\frac{1}{4} \hat{\mathrm{Y}}\left[(1-\hat{\mathrm{R}})^{2} U_{13} U_{2}+(1+\hat{\mathrm{R}})^{2} U_{2}^{-1} U_{13}\right] \\
=\frac{1}{4} \hat{\mathrm{Y}} \sigma_{1}\left[(1-\hat{\mathrm{R}})^{2}+(1+\hat{\mathrm{R}})^{2}\right]=\hat{\mathrm{Y}} \sigma_{1} .
\end{gathered}
$$

Eventually, on combining (19), (20), and (21):

$$
\begin{aligned}
U_{F G} C \sigma_{2} U_{F G}^{-1} & =C \sigma_{1} U_{F G} \sigma_{2} U_{F G}^{-1}=-\mathrm{i} C \hat{\mathrm{R}} \sigma_{3}, \\
U_{F G} D \sigma_{3} U_{F G}^{-1} & =D \sigma_{1} U_{F G} \sigma_{3} U_{F G}^{-1}=D \mathbb{1} .
\end{aligned}
$$

Therefore, the action of $U_{F G}$ summarized in Table 1 ensures that any $\hat{H}_{F G}$ of the Fulton-Gouterman type defined by (2), (3) can indeed be diagonalized in the spin subspace. The form of unitary transformed $\hat{\Pi}_{F G}$ and of operators $\hat{\mathrm{L}}_{ \pm}$ in (8) can be read off from Table 1 . Thereby the proof is completed.
Proof of Theorem 2. - If a hermitian operator $\hat{H}$ has the Fulton-Gouterman form (2), (3), then, according to Theorem 1, it is unitary equivalent to an operator diagonal in the spin subspace. Hence in order to prove Theorem 2 it suffices to show that the reverse holds, too.

A hermitian operator $\hat{H}$ is diagonal in the spin subspace if and only if $h_{1}=h_{2} \equiv 0$ in the expansion (11). Now any $h_{j} \neq 0$ in (11) can be decomposed as $h_{j}=\hat{\mathrm{X}}_{j}+\hat{\mathrm{Y}}_{j}$, where $\left[\hat{\mathrm{X}}_{j}, \hat{\mathrm{R}}\right]=0$ and $\left\{\hat{\mathrm{Y}}_{j}, \hat{\mathrm{R}}\right\}=0$, with $\hat{\mathrm{R}}$ being an arbitrary reflection operator. To this end, one takes

$$
\hat{\mathrm{X}}_{j}=\frac{1}{2}\left(h_{j}+\hat{\mathrm{R}} h_{j} \hat{\mathrm{R}}\right), \quad \hat{\mathrm{Y}}_{j}=\frac{1}{2}\left(h_{j}-\hat{\mathrm{R}} h_{j} \hat{\mathrm{R}}\right) .
$$

A unitary $U$ which commutes with any $\hat{\mathrm{X}}_{j}, j=0,3$, and brings a diagonal operator $\hat{H}$ into the Fulton-Gouterman form has to necessarily satisfy

$$
U \sigma_{3} U^{-1}=\sigma_{1} .
$$

At the same time, the transformed set $\left\{U \hat{\mathrm{Y}}_{0} \sigma_{0} U^{-1}, U \hat{\mathrm{Y}}_{3} \sigma_{3} U^{-1}\right\}$ has to become $\left\{\hat{\mathrm{Y}}^{\prime} \sigma_{2}, \hat{\mathrm{Y}}^{\prime \prime} \sigma_{3}\right\}$, where the set $\left\{\hat{Y}^{\prime}, \hat{Y}^{\prime \prime}\right\}$ is, modulo a possible sign change and multiplication by $\hat{\mathrm{R}}$ and a constant, equivalent to $\left\{\hat{Y}_{0}, \hat{Y}_{3}\right\}$. In conformity to the general rule $(*)$, the condition (23) fixes $U_{j k l}$ to be either $U_{F G}=U_{132}^{-1}$ or $U_{132}$. The first choice can be excluded in virtue of the second of eqs. (22). In the case of $U_{132}$, one finds with the help of identities (16)

$$
\begin{gathered}
U_{132} \sigma_{0} U_{132}^{-1}=\sigma_{0}, \quad U_{132} \sigma_{1} U_{132}^{-1}=\sigma_{3}, \\
U_{132} \sigma_{2} U_{132}^{-1}=-\hat{\mathbf{R}} \sigma_{2}, \quad U_{132} \sigma_{3} U_{132}^{-1}=\hat{\mathbf{R}} \sigma_{1},
\end{gathered}
$$

which is consistent with eqs. (20). Because $U_{j k}$ is symmetric in its indices, one can always adopt the convention that, when calculating the products $U_{j k} U_{l}^{-1}=U_{l} U_{j k}$ with unequal $j, k, l$, the indices are ordered such that $\epsilon_{j k l}=1$. With the above convention

$$
U_{j k} U_{l}^{-1}=U_{l} U_{j k}=\sigma_{j},
$$

i.e., $U_{31} U_{2}^{-1}=U_{2} U_{31}=\sigma_{3}$, and one finds [cf. eq. (21)]

$$
U_{132} \hat{Y} U_{132}^{-1}=\hat{Y} \sigma_{3} .
$$

Eventually, in virtue of identities (19), (24), (25),

$$
\begin{gathered}
U_{132} \hat{\mathrm{Y}} \sigma_{0} U_{132}^{-1}=\hat{\mathrm{Y}} \sigma_{3}, \quad U_{132} \hat{\mathrm{Y}} \sigma_{1} U_{132}^{-1}=\hat{\mathrm{Y}} \mathbb{1}, \\
U_{132} \hat{\mathrm{Y}} \sigma_{2} U_{132}^{-1}=\mathrm{i} \hat{\mathrm{Y}} \hat{\mathrm{R}} \sigma_{1}, \quad U_{132} \hat{\mathrm{Y}} \sigma_{3} U_{132}^{-1}=i \hat{\mathrm{Y}} \hat{\mathrm{R}} \sigma_{2} .
\end{gathered}
$$

Therefore, the unitary transformation induced by $U_{132}$ transforms the set $\left\{\left(\hat{\mathrm{X}}_{0}+\hat{\mathrm{Y}}_{0}\right) \sigma_{0},\left(\hat{\mathrm{X}}_{3}+\hat{\mathrm{Y}}_{3}\right) \sigma_{3}\right\}$ into $\left\{\hat{\mathrm{X}}_{0} \sigma_{0}, \hat{\mathrm{X}}_{3} \hat{\mathrm{R}} \sigma_{1}, \mathrm{i} \hat{\mathrm{Y}}_{3} \hat{\mathrm{R}} \sigma_{2}, \hat{\mathrm{Y}}_{0} \sigma_{3}\right\}$, thereby yielding the Fulton-Gouterman form (2), (3). The proof is completed.

Conclusions. - The respective sets of $2 \times 2$ hermitian operators of the Fulton-Gouterman type and those diagonal in the spin subspace were shown to be unitary equivalent. As an example, discrete parity $\mathbb{Z}_{2}$ symmetry of a two 
parameter extension of the Rabi model which smoothly interpolates between the latter and the Jaynes-Cummings model, the so-called generalized Rabi model (GRM), and of the two-photon and the two-mode quantum Rabi models was shown to enable their diagonalization in the spin subspace. The demonstrated diagonalized representation is expected to greatly simplify the description of time evolution and dissipative dynamics of the models. In the case of the GRM, supersymmetry on certain submanifolds in a parameter space has been established by Gritsev et al. 6. The diagonalized representation could facilitate here a much straightforward identification of supercharges by halving the dimensions of matrices involved.

An intimate relation of the generalized Rabi models with the class of differential operators of Dunkl type was established. Hopefully, this will help to address computational issues more efficiently. Many problems involving parity symmetry appear as potential candidates of further examples where one could encounter the Dunkl type operators. The diagonalization can be straightforwardly extended to spin $s>1 / 2$ models which possess an Abelian symmetry of the order of $N=2 s+1$ [30, 38. However the relation with the Dunkl type operators seems to be particular for spin $s=1 / 2$ models: for $N>2$ the Dunkl type operators are associated, in general, to nonabelian Coxeter groups [31].

The well known level-statistics criteria which have been applied with great success to autonomous particle systems are not applicable to the generalized Rabi models. The nearest-neighbour distribution of levels is not of the general type associated with chaotic systems and does not offer any conclusive evidence for quantum nonintegrability 39. Only the analysis of two-dimensional patterns of quantum invariants $\left\{\left(\epsilon_{n},\langle T\rangle_{n}\right)\right\}$ yields an unambiguous answer here. Braak's definition of integrability was shown not only to contradict the earlier pattern studies by Müller et al. 2, 2.3] but also to imply that any physically reasonable differential operator of Fulton-Gouterman type (i.e. leading to a TTRR) is integrable. This suggests that Braak's definition of integrability is most probably a faulty one. This is supported by the conclusions of ref. 40, that the Rabi model is not Yang-Baxter integrable.

$$
* * *
$$

Continuous support of MAKM and discussions with B. M. Rodríguez-Lara and A. Zhedanov are gratefully acknowledged.

References

[1] Fulton R. L. and Gouterman M., J. Chem. Phys., 35 (1961) 1059.

[2] Cibils M., Cuche Y. and Müller G., Z. Phys. B Cond. Mat., 97 (1995) 565.

[3] Stepanov V. V., Müller G. and Stolze J., Phys. Rev. E, 77 (2008) 066202.
[4] Schiró M., Bordyuh M., Öztop B. and Türeci H. E., Phys. Rev. Lett., 109 (2012) 053601.

[5] Tomka M., El Araby O., Pletyukhov M. and GritSEv V., Phys. Rev. A, 90 (2014) 063839.

[6] Tomka M., Pletyukhov M. and Gritsev V., Sci. Rep., 5 (2015) 13097.

[7] Xie Q.-T., Cui S., CaO J.-P., Amico L. and Fan H., Phys. Rev. X, 4 (2014) 021046; Zhang G. and Zhu H., Sci. Rep., 5 (2015) 8756.

[8] Zhang Y.-Z., J. Math. Phys., 54 (2013) 102104.

[9] Bargmann V., Comm. Pure Appl. Math., 14 (1961) 187.

[10] Barut A. O. and Girardello L., Commun. Math. Phys., 21 (1971) 41.

[11] Jaynes E. T. and Cummings F. W., Proc. IEEE, 51 (1963) 89.

[12] Rabi I. I., Phys. Rev., 49 (1936) 324; ibid, 51 (1937) 652.

[13] Schweber S., Ann. Phys. (N.Y.), 41 (1967) 205.

[14] Shore H. B. and Sander L. M., Phys. Rev. B, 7 (1973) 4537.

[15] Kus M., J. Math. Phys., 26 (1985) 2792.

[16] Schmutz M., J. Phys. A: Math. Gen., 19 (1986) 3565.

[17] Tur E. A., Opt. Spectrosc., 89 (2000) 574.

[18] BraAk D., Phys. Rev. Lett., 107 (2011) 100401.

[19] Moroz A., arXiv:1205.3139 Europhys. Lett., 100 (2012) 60010.

[20] Moroz A., Ann. Phys. (N.Y.), 338 (2013) 319; ibid 340 (2014) 252

[21] Moroz A., J. Phys. A: Math. Theor., 47 (2014) 495204.

[22] Chilingaryan S. A. and Rodríguez-Lara B. M., J. Phys. A: Math. Theor., 46 (2013) 335301.

[23] Khitrova G., Gibbs H. M., Kira M., Koch S. W. and SCherer A., Nature Phys., 2 (2006) 81.

[24] Bourassa J. et al., Phys. Rev. A, 80 (2009) 032109.

[25] Forn-Díaz P. et al., Phys. Rev. Lett., 105 (2010) 237001.

[26] NiemczyK T. et al., Nature Phys., 6 (2010) 772.

[27] Casanova J. , Romero G., Lizuain I., García-Ripoll J. J. and Solano E., Phys. Rev. Lett., 105 (2010) 263603.

[28] Erlingsson S. I., Egues J. C. and Loss D., Phys. Rev. $B, 82$ (2010) 155456.

[29] Galitski V. and Spielman I. B., Nature, 494 (2013) 49.

[30] Wagner M., J. Phys. A: Math. Gen., 17 (1984) 2319.

[31] Dunkl C. F., Canad. J. Math., 43 (1991) 1213.

[32] Vinet L. and Zhedanov A., Sigma, 7 (2011) 020.

[33] Manning M. F., Phys. Rev., 48 (1935) 161.

[34] Moroz A., Ann. Phys. (N.Y.), 351 (2014) 960.

[35] Fu L. and Hochstadt H., J. Math. Anal. Appl., 47 (1974) 162.

[36] Bohigas O., Giannoni M. J. and Schmit C., Phys. Rev. Lett., 52 (1984) 1.

[37] Peres A., Phys. Rev. Lett., 53 (1984) 1711.

[38] Zhang Y.-Z., Ann. Phys. (N.Y.), 347 (2014) 122.

[39] Kus M., Phys. Rev. Lett., 54 (1985) 1343.

[40] Batchelor M. T. and Zhou H.-Q., Phys. Rev. A, 91 (2015) 053808. 